\title{
Analysis of the damage effect of femtosecond-laser irradiation on extreme ultraviolet Mo/Si multilayer coating
}

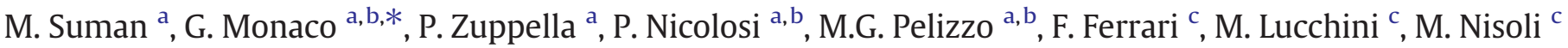 \\ a National Research Council-Istituto di Fotonica e Nanotecnologie, IFN-CNR, via Trasea 7, Padova, 35131, Italy \\ b Information Engineering Department, University of Padova, via Gradenigo 6B, Padova, 35131, Italy \\ c Politecnico di Milano, Department of Physics and Istituto di Fotonica e Nanotecnologie, IFN-CNR, Piazza L. da Vinci 32, 20133 Milano, Italy
}

\section{A R T I C L E I N F O}

\section{Article history:}

Received 21 December 2010

Received in revised form 31 August 2011

Accepted 31 August 2011

Available online 6 September 2011

\section{Keywords:}

Extreme UV lithography

Multilayers

Attosecond physics

Laser damage

\begin{abstract}
A B S T R A C T
Damage analysis of Mo/Si multilayer coatings exposed to fs infrared laser irradiation has been performed. The Mo/Si a-periodic multilayer samples were specifically designed with wide reflectivity bandwidth and suitable phase chirp in order to reflect attosecond pulses.

After irradiation, the mirror surface was analyzed by using an optical microscope and a profilometer. The stoichiometry of the compounds formed at the sample surface after the irradiation was investigated using X-Ray photoemission spectroscopy. The performances of the irradiated samples with the reflected pulse characteristics have been derived via reflectivity and phase measurements.
\end{abstract}

(c) 2011 Elsevier B.V. All rights reserved.

\section{Introduction}

Extreme Ultraviolet (EUV) Multilayer (ML) coatings are applied in the extreme ultraviolet spectral region in order to get high optical performance, otherwise not obtainable with standard optical systems. EUV reflective ML optics have been extensively studied and are applied in space instruments, synchrotron radiation (SR) facilities and in the extreme ultraviolet projection lithography systems technique that are designed to extend the resolution limit well beyond the limit presently achievable with a lithographic apparatus. Furthermore, EUV-ML are more recently actively studied for application with the EUV and Soft X-ray Free Electron Laser (EUV-FEL) systems and in High order Harmonic Generation (HHG) experiments to produce attosecond pulses. The design of suitable multilayer optics for attosecond technology has been studied in the last years $[1,2]$ and a number of authors have investigated the possibility to temporarily preserve or compress attosecond pulses through phase compensation $[3,4]$ since attosecond pulses produced by HHG present an intrinsic chirp [5], which has to be compensated in order to obtain nearly transformlimited pulses. In this context, it is of great interest the study of the radiation induced damage on ML structures by the interaction with ultra-short laser pulses. This, in particular, can have wide impact on several applications characterized by extreme operational conditions due to high fluencies and short pulse duration, like for examples in

\footnotetext{
* Corresponding author at: National Research Council-Institute for Photonics and Nanotechnology, via Trasea 7, Padova, 35131 Italy.

E-mail address: monaco.gianni@gmail.com (G. Monaco).
}

the case of $\mathrm{x}$-ray laser cavities, synchrotron radiation focusing, laser produced plasmas radiation applications, HHG based experiments.

The laser-matter interaction mechanism depends strongly on the optical and thermodynamic properties of the target material, the laser wavelength, and pulse duration and energy. Several experiments have been performed concerning the interaction of ultrashort laser pulses with materials. In particular the damage induced for example on metals $[6,7]$, dielectrics [7,8], semiconductors [9], thin films [10,11], ML $[7,12,13]$ has been studied for a wide range of experimental conditions. Nolte et al. [14], have studied the interaction process of ultrashort laser pulses, ranging from 150 fs to $30 \mathrm{ps,} \mathrm{with} \mathrm{metals,} \mathrm{explaining} \mathrm{the} \mathrm{exper-}$ imental results with a two-temperature based model. Jeschke et al. [15] have calculated, with a model based on molecular dynamic theory, the ablation threshold for Silicon with pulses at $780 \mathrm{~nm}$ of 20 and $500 \mathrm{fs}$, finding a good agreement with the experiment. More recently, damage analysis have been focalized on the interaction with high intensity EUV wavelength for future application in the next FEL facilities. Hau-Riege et al. [16] have investigated the interaction with low- $\mathrm{Z}$ materials $\left(\mathrm{SiC}, \mathrm{B}_{4} \mathrm{C}\right.$ and $\mathrm{Si}$ ) in order to retrieve their damage thresholds at $32.5 \mathrm{~nm}$ and then explored the materials behavior when exposed to laser beam at different wavelengths in the EUV [17]. Basically, the interaction process and consequent induced effects on the target can be described via excitation of electrons on the conduction band via impact and multiphoton ionization, subsequent heating of the electron plasma and coupling with energy transfer to the lattice. For ultrashort pulses (less than few ps duration) heat diffusion and melting are inhibited during the pulse and the consequent shock due to laser-matter interaction causes ablation. 
Laser damage induced on Mo/Si multilayer coatings with fs laser pulses has been studied in the low fluence regime by Hoeche et al. [13] by using cross-sectional Transmission Electron Microscope, to observe the modification induced by a $775 \mathrm{~nm}$ Ti:Sapphire laser of $120 \mathrm{fs}$ at $280 \mathrm{~mJ} / \mathrm{cm}^{2}$ of fluence in air, showing that the top-layers in the structure were completely intermixed after laser exposure. Mo/Si multilayers structures designed for EUV lithography have also been studied by exposure to high intensity EUV pulses at $13.5 \mathrm{~nm}$ at different fluence in order to determine the ablation threshold [18].

In this work we have studied how the fs laser pulses exposure in air modify the optical performance and surface compositional characteristics of Mo/Si ML mirrors.

The Mo/Si multilayer structure was specifically designed for the reflection of attosecond pulses, generated in an HHG experiment by driving pulses with a central wavelength of $800 \mathrm{~nm}$ [19]. The Mo/Si structure was designed to compress a positively chirped $\left(0.3-\mathrm{fs}^{2}\right.$ chirp), 450-as Gaussian pulse with a $30-\mathrm{eV}$ bandwidth (ranging from 80 to $110 \mathrm{eV}$ ) down to $120 \mathrm{as}$, as shown in Fig. 1. For this reason the ML structure is a-periodic and has a relatively wide reflectivity bandwidth with phase behavior to compensate for the frequency chirp of the incident pulse.

The multilayer mirror was exposed to intense 25-fs, $800-\mathrm{nm}$ pulses (at $1-\mathrm{kHz}$ repetition rate) by varying intensity and exposure time. A first damage threshold estimation was based on visual inspection at microscope and profilometry; the material composition at impinged areas was determined through X-ray Photoemission Spectroscopy (XPS);
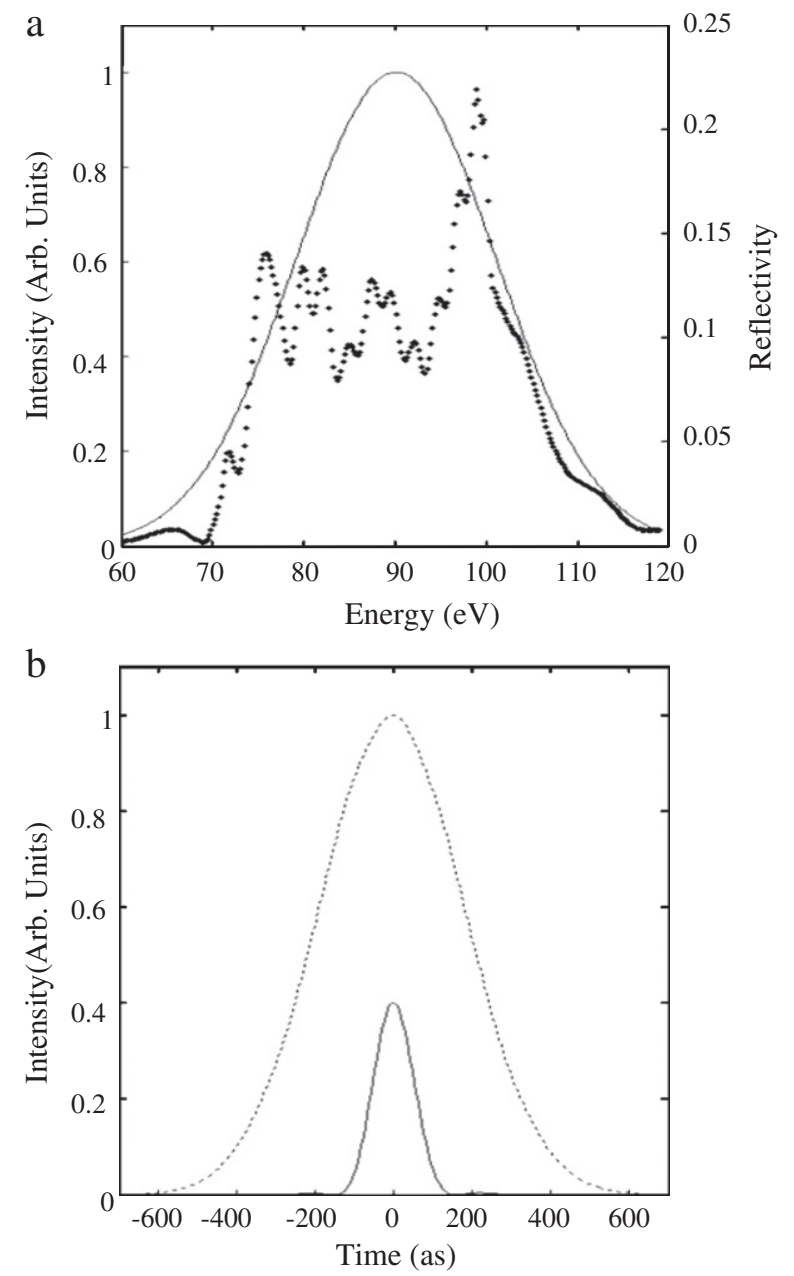

Fig. 1. a) In continuous line the theoretical reflectivity of the considered multilayer and in dotted line the intensity of the incident Gaussian pulse. b) In continuous line the pulse reflected using the theoretical reflection and in dotted line the incident pulse.
Optical performances of the ML structure have been derived from reflectivity and phase measurements, and these results have been used to predict the reflected pulse characteristics. Reflectivity and total electron yield measurements have been performed at the BEAR beamline [20-22] of the ELETTRA synchrotron.

\section{Experimental details}

25-fs, $800-\mathrm{nm}$ pulses with an energy up to $0.7 \mathrm{~mJ}$ and $1-\mathrm{kHz}$ repetition rate have been generated by a Ti:sapphire laser system. The IR pulses were focused by using a 3.5-m radius of curvature mirror on the multilayer mirror, which was placed at $1 \mathrm{~m}$ from the focusing mirror, in order to mimic the conditions of a realistic experimental setup. The pulse intensity profile at the mirror position was acquired with a beam profiler and the laser spot was estimated to be $822 \times 1230 \mu \mathrm{m}^{2}$ at $1 / \mathrm{e}^{2}$ of the peak value. During the experiment only a fraction of the laser pulse energy, well below the ablation threshold, corresponding to a fluence of $42.5,17.5$ and $5 \mathrm{~mJ} / \mathrm{cm}^{2}$ was used. The sample was impinged in nine different points, varying the intensities and the exposure times. The experiment was carried on in air. Fig. 2 shows the map of the impinged points of the multilayer mirror, with the indication of the parameters used in the experiment, namely the pulse energies and the exposure times. For comparison, point 0 (hereafter called reference point), which was not irradiated, was also analyzed. First, we irradiated points 1 to 3 with an exposure time of $60 \mathrm{~s}$ and an increasing laser fluence. At a visual observation, only point 3 appeared visibly damaged. Therefore we irradiated points 4 to 9 with the same laser fluence as point 3 but with shorter exposure times, in order to determine the exposure time threshold.

\section{Results and discussion}

Point 3 to 9 were inspected with an optical microscope, which highlighted a black spot in points 3 and 9 and a white spot in the other impinged points (Fig. 3 ). This different appearance can be justified by a carbonization of the sample in points 3 and 9 . The sample surface was mapped by a "KLA Tencor P-16+" profilometer: only in correspondence of points 3 and 9 a circular bases bump can be noticed, with a diameter of about 420 and $100 \mu \mathrm{m}$ respectively, as shown in Fig. 4.

References [12] and [15] report on experiments of surface damage induced by femtosecond laser pulses of duration of 25 and $400 \mathrm{fs}$ and $130 \mathrm{fs}$. The target were in one case a multilayer structure for reflecting a wavelength close to the laser one, in the second Silicon n-doped; both experiments have been performed in air. Although the targets

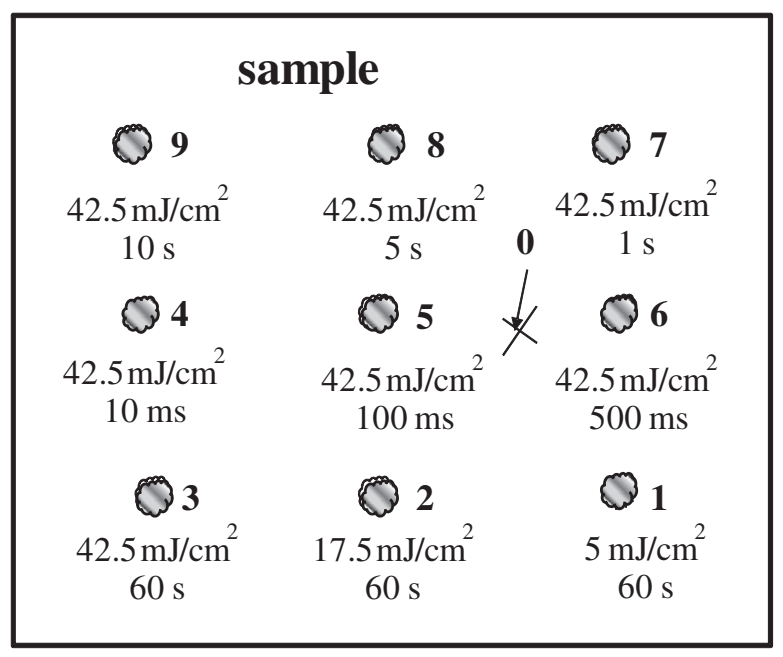

Fig. 2. Schematic representation of the sample in the region exposed to IR pulses, with the indication of irradiation time and fluence $\left(\mathrm{J} / \mathrm{cm}^{2}\right)$. "0" is an unstriked point. 

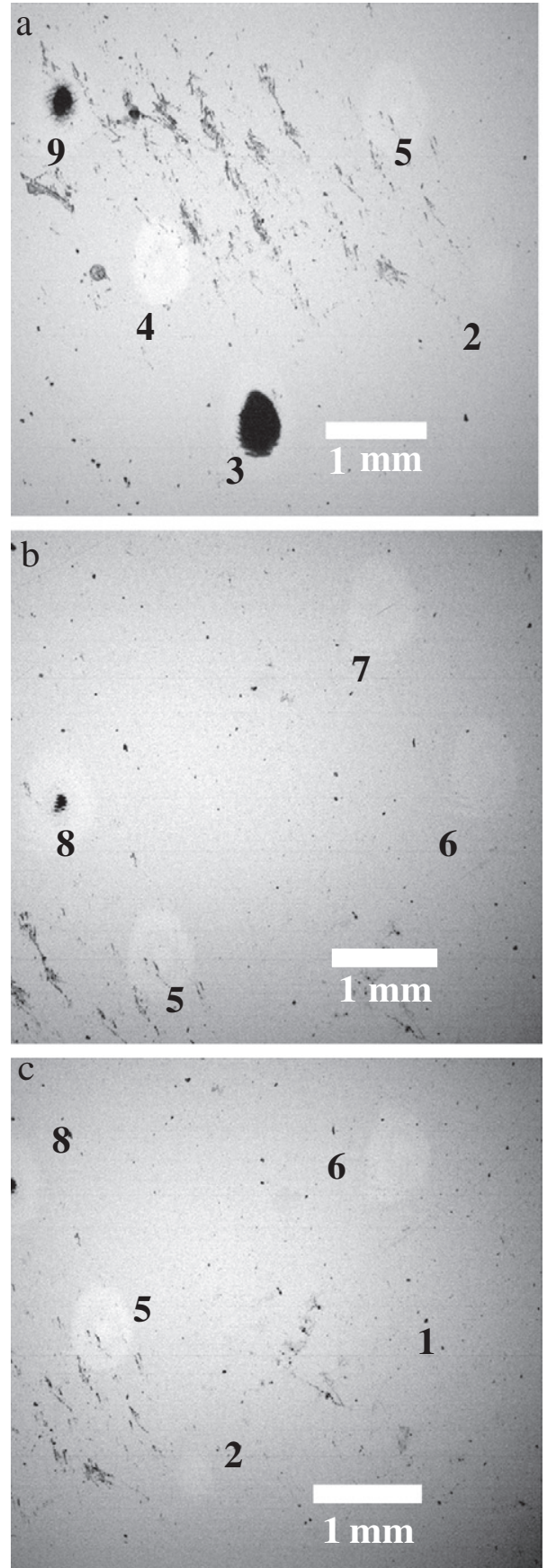

Fig. 3. Images of the irradiated multilayer surface obtained by using an optical microscope. Points 9, 4, 3, 5, 2 (3a.), points 8, 5, 7, 6 (3b.), and point 5,2,6, 1 (3c.).

are different from our Mo/Si multilayer the results reported in these papers are indeed interesting to addressing the problem about the value of the ablation threshold. In both papers the derived ablation threshold are considerably higher than the max fluence in our experiment. In addition in [9] the ultra-fast melting threshold of Silicon for fs laser pulses has been estimated to be slightly higher than $10 \mathrm{~mJ} / \mathrm{cm}^{2}$. Based on these results we conclude we should be in a non-equilibrium regime of non-thermal melting at least in the two cases of highest fluencies.

Due to the high absorption coefficient of molybdenum at $800 \mathrm{~nm}$, we have calculated that more than $80 \%$ of the pulse energy absorbed by the coated mirror is absorbed by the first ten molybdenum layers: for this reason we can assume that a possible material structural change has to involve only the first layers near the surface of the multilayer coating. Therefore, in order to analyze the effects of laser

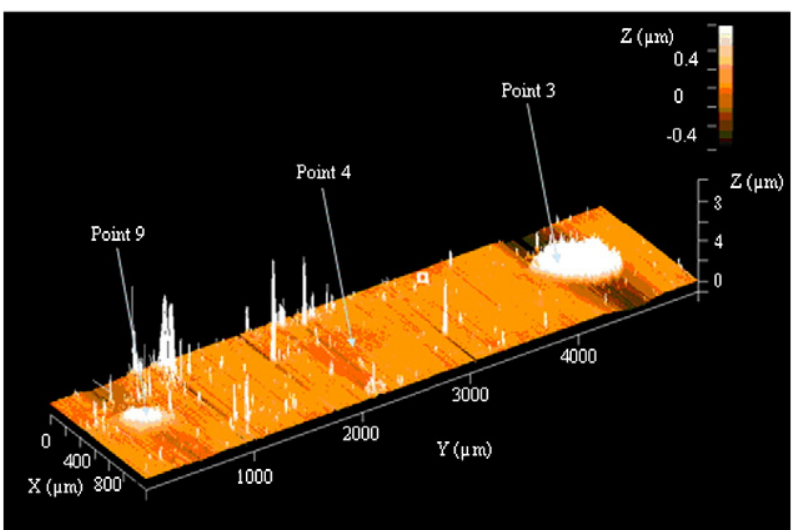

Fig. 4. The topography of the multilayer surface near points 3, 4 and 9 obtained by a "KLA Tencor P-16+" profilometer. Correspondingly, points 3, 4 and 9 are signed.

irradiation we decided to investigate the chemical composition at the surface of the irradiated points by means of X-ray photoemission spectroscopy (XPS) technique. The XPS investigation was performed at the ELETTRA synchrotron, BEAR beamline. The analysis of the damaged points 4 and 9 and of the reference point was carried out placing the sample at $10^{\circ}$ of incidence with respect to the incoming photons. The photo-emitted electrons were collected placing the electron analyzer along the direction perpendicular to the sample surface. Fig. 5 shows the XPS spectra obtained by using $650 \mathrm{eV}$ radiation. Such a photon energy is sufficient to excite the core electrons of all the elements expected to be present on the multilayer surface: Molybdenum, Carbon, Silicon and Oxygen. The spectra clearly show how Molybdenum comes out on the sample surface after the laser irradiation: the peak of Mo 3d (labeled as F in Fig. 5) is clearly visible for point 4 and point 9 , but there is no evidence for the reference point.

We have then carried out a detailed analysis of the emission peaks of Carbon, Oxygen and Silicon at different surface points. The kinetic energy (KE) of the photo-emitted electron is related to the energy of the impinging photon, $h v$, through the following relationship:

$\mathrm{KE}=h v-\mathrm{E}_{\mathrm{b}}-\psi$

where $E_{b}$ is the binding energy of the photo-emitted electron [23] and $\psi$ is the work function of the spectrometer. XPS was carried out with the same experimental apparatus used for the previous overview spectra,

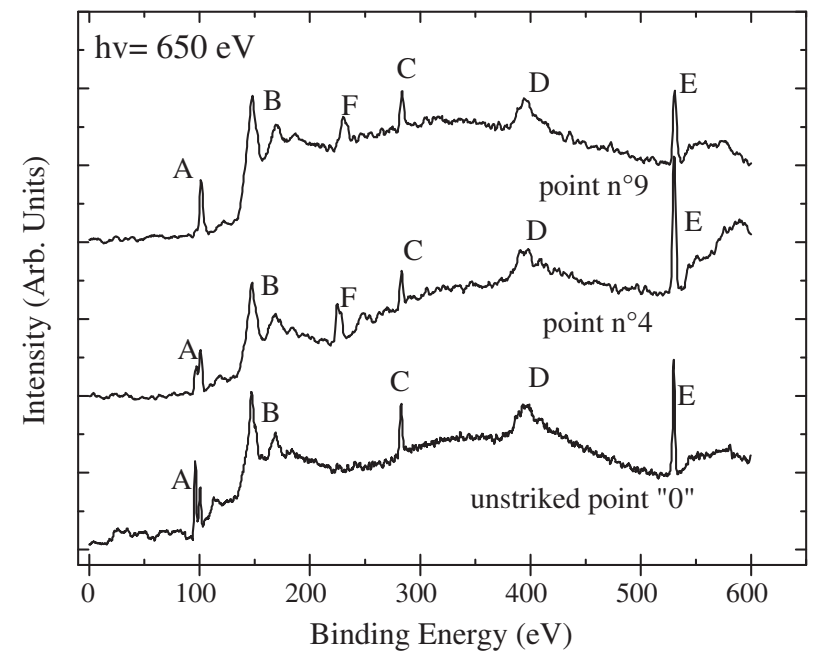

Fig. 5. Overview of the photo-emitted electrons from three different points of the sample (points 0, 4 and 9 from bottom to top), with an impinging photon energy of $\mathrm{h} v=650 \mathrm{eV}$. The different marked peaks correspond to: $\mathrm{A}=\mathrm{Si} 2 \mathrm{p}$ XPS; $\mathrm{B}=\mathrm{O}$ KVV in $\mathrm{SiO}_{2}$ Auger; $\mathrm{C}=\mathrm{C}$ 1s XPS; $\mathrm{D}=\mathrm{C}$ KLL Auger; $\mathrm{E}=\mathrm{O}$ 1s XPS; $\mathrm{F}=$ Mo 3d XPS 
Table 1

Compositional analysis of Carbon, Oxygen and Silicon on the multilayer surface.

\begin{tabular}{lllllll}
\hline & Point 0 & Point 3 & Point 4 & Point 9 & Point 2 & Point 6 \\
\hline $\mathrm{Si}$ & 0.5 & 0.6 & 0.4 & 0.4 & 0.5 & 0.4 \\
$\mathrm{O}$ & 0.2 & 0.2 & 0.3 & 0.3 & 0.2 & 0.3 \\
$\mathrm{C}$ & 0.3 & 0.2 & 0.3 & 0.3 & 0.3 & 0.3 \\
\hline
\end{tabular}

but changing the energies of the impinging photons in order to obtain photo-emitted electrons with the same kinetic energy for all the different elements, to eliminate spurious effects due to detector efficiency, electron analyzer transmission and electron mean free path [24]. In this way, the same narrow energy window $(90-100 \mathrm{eV})$ was set for the electron analyzer, which exhibits the maximum transmittance in such an energy range. Such a methodology allowed us a direct comparison between the experimental data, which can be used to understand the nature of the chemical bonds and the surface stoichiometry. The $\mathrm{Si}$ 2p analysis, shown in Fig. 6 was carried out for points 3, 4, 6 and 9. Fig. 6 shows that Silicon is mainly oxidized (peak around $104 \mathrm{eV}$ ) in all the analyzed points, but with different yields, as it is also shown by the peaks at $99.4 \mathrm{eV}$. The limited resolution of the instrument $(0.1 \mathrm{eV})$ does not allow us to clearly understand if the $99.4 \mathrm{eV}$ peak is due to $\mathrm{Si}-\mathrm{Si}$ bonds or to $\mathrm{Mo}-\mathrm{Si}$ bonds [25], the latter indicating the formation of Molybdenum Silicide $\left(\mathrm{MoSi}_{2}\right)$. This peak is not very pronounced for points 4 and 6, disappears for point 9 and is very marked for point 3 .

Such experimental results suggest the following physical scenario for the laser-mirror interaction: (i) as a first step, the IR irradiation favors the oxidation of the Silicon reducing the yield of $\mathrm{Si}-\mathrm{Si}$ bonds (points 4 and 6); (ii) upon increasing the laser exposure, the Silicon oxidizes completely (point 9 ) and, as a final step (iii) a further increase of the exposure time (point 3 ) induces material from the inside of the multilayer to come out on the sample surface, bringing some clean Silicon atoms from the inner layers. This picture is confirmed by the stoichiometry reported in Table 1: the reported values represent the ratio between the peak area of each element and the sum of the areas of all peaks, where each area was obtained correcting the data for their photo-ionization cross section [24]. The table shows that the $\mathrm{Si}$

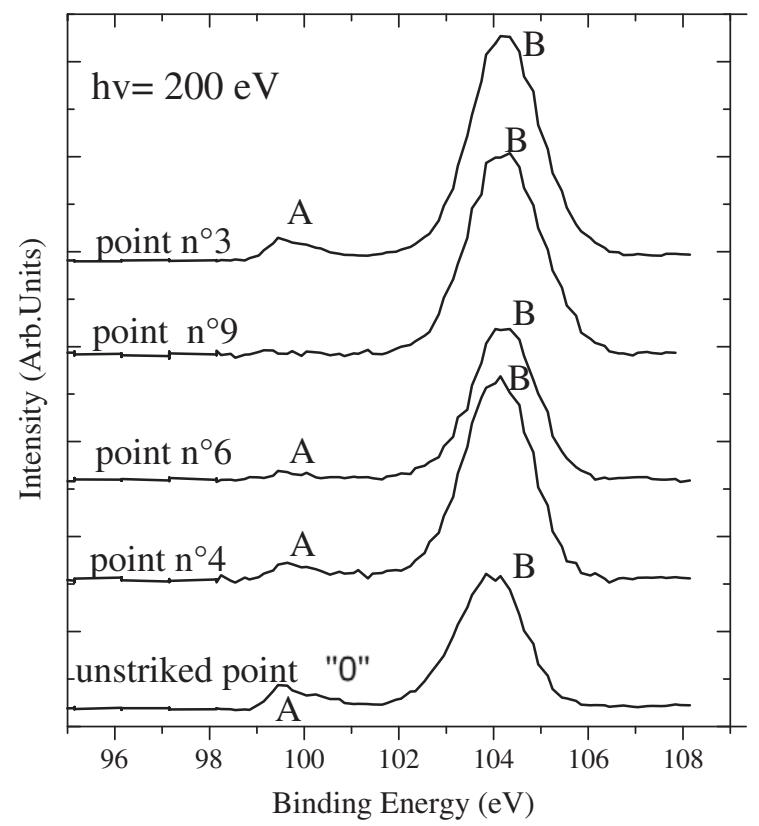

Fig. 6. XPS of Si 2p electrons emitted from different points of the sample (points 0, 4, 6, 9 and 3 from bottom to top). The Silicon on the surface is mainly oxidized regardless of the number of laser shots at the maximum fluence of $42.5 \mathrm{~mJ} / \mathrm{cm}^{2}$. The peak "B" around $104 \mathrm{eV}$ comes from $\mathrm{SiO}_{\mathrm{x}}$ bonds, while the peak at " $\mathrm{A}$ " $99.4 \mathrm{eV}$ can be both due to pure $\mathrm{Si}$ bonds or to $\mathrm{Mo}-\mathrm{Si}$ bonds [22]. content for point 3 is higher than for points 4, 6 and 9 and is even higher than for point 0 . Furthermore, the stoichiometry of point 2 may indicate that the laser-surface interaction process has a threshold, because in this case no modification in the sample stoichiometry emerges between points 0 and 2 .

As a final consideration, even if the instrument resolution is not enough to distinguish between pure $\mathrm{Si}-\mathrm{Si}$ bonds and $\mathrm{Mo}-\mathrm{Si}$ bonds, the analysis of the Si $2 \mathrm{p}$ peak can also give information about the $\mathrm{MoSi}_{2}$ formation: if any Mo-Si would have formed and emerged on the surface, we should see the $99,4 \mathrm{eV}$ peak in the analysis of point 4 and point 9 , on the contrary such a peak disappears, thus leading to the interpretation that Molybdenum just comes out from the inner structure (Fig. 5) during the laser irradiation, without forming any chemical bond with Silicon.

Reflectivity and phase behavior of the Mo/Si multilayer were also measured at the BEAR beamline. In particular, the phase behavior was obtained recovering the standing wave distribution obtained from total electron yields (TEY), $\eta_{\mathrm{T}}$, photoemission measurements:

$\eta_{\mathrm{T}}(E)=\mathrm{C}(E) \cdot I_{0} \cdot(1+R(E)+2 \cdot \sqrt{R(E)} \cdot \cos \Phi(E))$

where $C(E)$ takes into account the material dependence of the TEY data, $I_{0}$ is the intensity of the incident radiation, $R(E)$ is the reflectivity and $\Phi$ is the multilayer reflectivity phase [26]. In order to obtain the multilayer reflectivity phase behavior from the TEY data the $C(E)$ term has to be recovered: in our case we used a method based on TEY measurement at two slightly different incidence angles $20 \mathrm{deg}$ and $20.5 \mathrm{deg}$ as reported in Ref. [27]. Such a method offers great advantages since it is not sensitive to the change of the material at the sample surface, which can be induced by laser irradiation.

The reflectivity of the sample has been measured using an incident angle of $20^{\circ}$, the detector was a Si photodiode and the TEY data were collected by using a Keithley 6517A current amplifier, with a collection voltage of $20 \mathrm{~V}$, which induces a saturation of the TEY signal. The multilayer was optimized for an s-polarized incident beam while the finite dimension of the exit slit in the synchrotron beamline does not allow the measurement with a fully linearly polarized radiation. For this reason the aperture of the entrance slit of the polarization selector placed at the beginning of the beamline was set in order to minimize the angular acceptance, so to get a polarization degree of about $\mathrm{I}_{\mathrm{pol}}=0.8$ and still a good signal. Above $80 \mathrm{eV}$ the reflectivity measured with $\mathrm{I}_{\mathrm{pol}}=0.8$ is attenuated by a constant product factor; below $80 \mathrm{eV}$ the uncertainty in the polarization degree could lead to a maximum error of $2.5 \%$ at $71.5 \mathrm{eV}$ in reflectivity. On the contrary, phase recovery is almost not affected by the polarization degree uncertainty. We estimate an uncertainty of about $5 \%$ for the reflectivity. The spot of the beam used at BEAR $\left(100 \times 350 \mu \mathrm{m}^{2}\right)$ is smaller than the one used during the irradiation.

The results obtained from the TEY data allow us to obtain interesting information on the performances of the multilayer structures. The TEY data, in fact, depend on the standing wave behavior and, consequently, on the reflectivity and phase behavior (see Fig. 7): a change in the frequency of oscillations, for example, reflects a change in the chirp of the multilayer reflectivity phase.

The reflectivity signal in the case of points 3 and 9 was too low and the TEY data oscillations were comparable to the TEY data uncertainty: the results obtained were therefore considered not meaningful. To show the effect of the laser exposure on the multilayer we have reported in Fig. 8a) and b) the reflectivity and the experimental TEY data of the first set of points ( 1 to 3 ), irradiated with a constant exposure time (see Fig. 2). Point 0 is also shown as a reference. The plotted TEY data are then normalized to a beam intensity monitor signal. The beam monitor is a gold mesh which is placed after the beamline monochromators and the drain current signal from the mesh, due to photoelectric effect, is proportional to the beam intensity. In this way TEY 


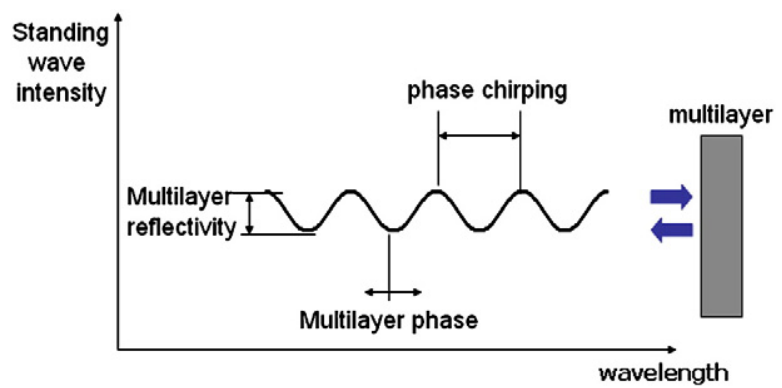

Fig. 7. Schematic of the standing wave parameters affected by the multilayer performances

data, which are not normalized to $C(E)$, can give a qualitative representation of the multilayer changes at the uppermost layer which have been accounted in the previous XPS analysis.

Fig. 8a) and b) report that point 1 presents similar behavior with respect to point 0 . On the contrary, radiation impinging point 2 is reflected with an $85 \%$ narrower bandwidth and there is a change in the frequency of the oscillations of the TEY data for this point.

Fig. 9a) and b) show respectively the reflectivity and the TEY data of the second set of points ( 3 to 9 ), irradiated with a constant laser fluence. Point 0 is shown again as a reference. The reflectivity drops as a function of the exposure time (Fig. 9a)), and this drop is more marked in the 90-100 eV spectral region. A constant enhancement of the TEY signal is therefore observed as a function of the number of pulses impinging the sample. In particular there is an enhancement of two peaks, one at about $107.5 \mathrm{eV}$ (absorption of $\mathrm{SiO}_{2}$ [22]) and the other at about $115 \mathrm{eV}$. The intensity of the peak at $107.5 \mathrm{eV}$ and the smaller peak at $105 \mathrm{eV}$ depends on the growth of the $\mathrm{SiO}_{2}$ at the sample surface, as described before.

Reflectivity curve and TEY data normalized to the beam intensity and the $C(E)$ factor [27] at the different points were used to simulate the reflection characteristics of the mirror assuming a 450-as Gaussian
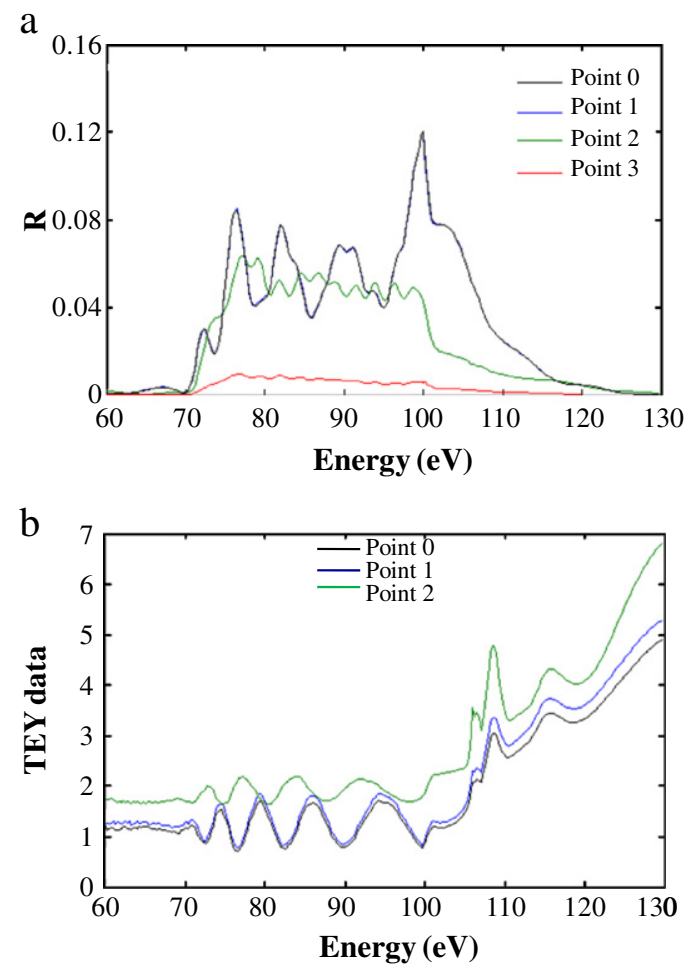

Fig. 8. Reflectivity, case a), and TEY data, case b), for points 1 to 3 , compared to the reference point 0 .
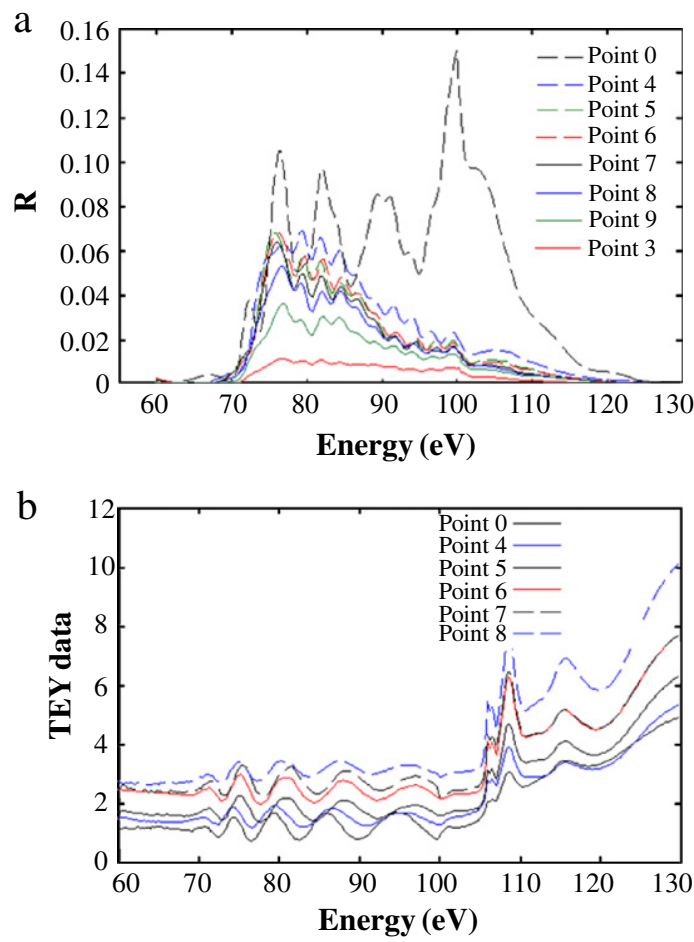

Fig. 9. Reflectivity, case a), and TEY data, case b), for points 3 to 9, compared to the reference point 0 .

pulse with positive chirp incident on the mirror. The results are shown in Table 2, where respectively efficiency loss, normalized to that one of the un-exposed area, and time duration of the reflected pulse are reported. Analyzing such results we can conclude that:

(i) if the Mo/Si multiplayer is irradiated by $5 \mathrm{~mJ} / \mathrm{cm}^{2}$ femtosecond pulses at $1 \mathrm{kHz}$ repetition rate and for an exposure time of $60 \mathrm{~s}$ the performances are only slightly changed;

(ii) if the Mo/Si multilayer is irradiated by $17.5 \mathrm{~mJ} / \mathrm{cm}^{2}$ femtosecond pulses at $1 \mathrm{kHz}$ repetition rate and for an exposure time of $60 \mathrm{~s}$ the performances are changed but they can still be considered as appropriate;

(iii) if the Mo/Si multilayer is irradiated by $25 \mathrm{~mJ} / \mathrm{cm}^{2}$ femtosecond pulses at $1 \mathrm{kHz}$ repetition rate and for an exposure time of $10 \mathrm{~s}$ the reflectivity is completely altered, affecting the efficiency of the optics.

In any case, when the irradiation induces only the oxidation of silicon at the sample surface (points 4 to 9), the multilayer structure still behave as a pulse time compressor: in fact, as shown in Table 2, the time duration of the reflected pulse is always shorter than 200 attoseconds.

Table 2

Intensity peak and time duration of the reflected pulses calculated using the experimental data obtained in the different points and an ideal incident Gaussian pulse.

\begin{tabular}{lll}
\hline Point \# & Intensity peak (Arb. units) & Time duration \\
\hline 0 & 1 & 134 attoseconds \\
1 & 0.95 & 141 attoseconds \\
2 & 0.8 & 135 attoseconds \\
3 & - & - \\
4 & 0.36 & 191 attoseconds \\
5 & 0.3 & 189 attoseconds \\
6 & 0.29 & 196 attoseconds \\
7 & 0.37 & 140 attoseconds \\
8 & 0.3 & 160 attoseconds \\
9 & - & - \\
\hline
\end{tabular}




\section{Conclusions}

A Mo-Si multilayer sample designed to reflect and compress isolated attosecond pulse was exposed to an intense 25-fs, 800-nm Ti-Sa laser pulses at $1-\mathrm{kHz}$ repetition rate.

Intensity and exposure time were varied. Different structural and optical techniques have been used to evaluate the exposure effects. Both reflectivity and phase of the multilayer have been measured and the characteristics of the reflected pulses have been accordingly derived. Below $17.5 \mathrm{~mJ} / \mathrm{cm}^{2}$ no significant change in the multilayer performances has been observed, even for a time exposure of $60 \mathrm{~s}$. At $42.5 \mathrm{~mJ} / \mathrm{cm}^{2}$ a significant variation of reflectivity performances has been always observed, even for $10 \mathrm{~ms}$ exposure time. In particular experimental results suggest that at low intensity the IR irradiation induces the oxidation of the Silicon reducing the yield of $\mathrm{Si}-\mathrm{Si}$ bonds, upon increasing the laser exposure, the Silicon oxidizes completely and, for a further increase of the exposure time the material from the inside of the multilayer comes out on the sample surface, bringing clean Silicon atoms from the inner layers.

\section{Acknowledgments}

This work has been supported by ASI grant n. I/015/07/0. The work has been performed also in the framework of the COST ACTION MP0601 "Short wavelength radiation sources". The authors acknowledge David Windt for the deposition of the multilayer sample; Angelo Giglia, Nicola Mahne and Stefano Nannarone of BEAR beam-line for support during measurements at ELETTRA Synchrotron Trieste and Matteo Meneghini for the measurements with the optical microscope. We acknowledge support from the European Research Council within the ERC grant agreement n. 227355 - ELYCHE, the European Union within contract n. 228334 JRA-ALADIN (Laserlab Europe II) and the support by the Marie Curie Research Training Networks ATTOFEL.

\section{References}

[1] I.L. Beigman, A.S. Pirozhkov, E.N. Ragozin, J. Opt. A 4 (2002) 433.

[2] A. Wonisch, T. Westerwalbesloh, W. Hachmann, N. Kabachnik, Thin Solid Films 464 (465) (2004) 473.

[3] A.-S. Morlens, P. Balcou, P. Zeitoun, C. Valentin, V. Laude, S. Kazamias, Opt. Lett. 30 (2005) 1554.
[4] A.-S. Morlens, R. López-Martens, O. Boyko, P. Zeitoun, P. Balcou, K. Varjú, E. Gustafsson, T. Remetter, A. L'Huillier, S. Kazamias, J. Gautier, F. Delmotte, M.-F. Ravet, Opt. Lett. 31 (2006) 1558.

[5] Y. Mairesse, A. de Bohan, L.J. Frasinski, H. Merdji, L.C. Dinu, P. Monchicourt, P. Breger M. Kovačev, R. Taïeb, B. Carré, H.G. Muller, P. Agostini, P. Salières, Science 302 (no. 5650) (2003) 1540.

[6] K. Furusawa, K. Takahashi, H. Kumagai, K. Midorikawa, M. Obara, Appl. Phys. A 69 (1999) S359.

[7] B.C. Stuart, M.D. Feit, S. Herman, A.M. Rubenchik, B.W. Shore, M.D. Perry, J. Opt. Soc. Am. B 13 (1996) 459.

[8] B.C. Stuart, M.D. Feit, A.M. Rubenchik, B.W. Shore, M.D. Perry, Phys. Rev. Lett. 74 (1995) 2248.

[9] A. Rousse, C. Rischel, S. Fourmaux, I. Uschmann, S. Sebban, G. Grillon, P. Balcou, E Förster, J.P. Geindre, P. Audebert, J.C. Gauthier, D. Hulin, Nature 410 (2001) 65.

[10] N. Yasumaru, K. Miyazaki, J. Kiuchi, Appl. Phys. A 76 (2003) 983.

[11] P. Ceccherini, M.-G. Pelizzo, P. Villoresi, S. De Silvestri, M. Nisoli, S. Stagira, Appl. Opt. 38 (1999) 21.

[12] J. Bonse, S. Baudach, W. Kautek, E. Welsch, J. Krüger, Thin Solid Films 408 (2002) 297.

[13] T. Höche, D. Ruthe, T. Petsch, Appl. Phys. A 79 (2004) 961.

[14] S. Nolte, C. Momma, H. Jacobs, A. Tünnermann, B.N. Chichkov, B. Wellegehausen, H. Welling, J. Opt. Soc. Am. B 14 (1997) 2716.

[15] H.O. Jeschke, M.E. Garcia, M. Lenzner, J. Bonse, J. Krüger, W. Kautek, Appl. Surf. Sci. 197-198 (2002) 839.

[16] S.P. Hau-Riege, R.A. London, R.M. Bionta, M.A. McKernan, S.L. Baker, J. Krzywinski, R. Sobierajski, R. Nietubyc, J.B. Pelka, M. Jurek, L. Juha, J. Chalupsky, J. Cihelka, V. Hajkova, A. Velyhan, J. Krasa, J. Kuba, K. Tiedtke, S. Toleikis, T. Tschentscher, H. Wabnitz, M. Bergh, C. Caleman, K. Sokolowski-Tinten, N. Stojanovic, U. Zastrau, Appl. Phys. Lett. 90 (2007) 173128.

[17] S.P. Hau-Riege, R.A. London, R.M. Bionta, D. Ryutov, R. Soufli, S. Bajt, M.A McKernan, S.L. Baker, J. Krzywinski, R. Sobierajski, R. Nietubyc, D. Klinger, J.B. Pelka, M. Jurek, L. Juha, J. Chalupsky, J. Cihelka, V. Hajkova, A. Velyhan, J. Krasa, K. Tiedtke, S. Toleikis, H. Wabnitz, M. Bergh, C. Caleman, N. Timneanu, Appl. Phys. Lett. 95 (2009) 111104

[18] A.R. Khorsand, R. Sobierajski, E. Louis, S. Bruijn, E.D. van Hattum, R.W.E. van de Kruijs, M. Jurek, D. Klinger, J.B. Pelka, L. Juha, T. Burian, J. Chalupsky, J. Cihelka, V. Hajkova, L. Vysin, U. Jastrow, N. Stojanovic, S. Toleikis, H. Wabnitz, K. Tiedtke, K. Sokolowski-Tinten, U. Shymanovich, J. Krzywinski, S. Hau-Riege, R. London, A. Gleeson, E.M. Gullikson, F. Bijkerk, Opt. Express 18 (2010) 700.

[19] M. Suman, F. Frassetto, P. Nicolosi, M.-G. Pelizzo, Appl. Opt. 46 (2007) 8159

[20] S. Nannarone, F. Borgatti, A. DeLuisa, B.P. Doyle, G.C. Gazzadi, A. Giglia, P. Finetti, N. Mahne, L. Pasquali, M. Pedio, G. Selvaggi, G. Naletto, M.G. Pelizzo, G. Tondello, AIP Conf. Proc. 705 (2004) 450.

[21] S. Nannarone, A. Giglia, N. Mahne, A. De Luisa, B. Doyle, F. Borgatti, M. Pedio, Pasquali, G. Naletto, M.G. Pelizzo, G. Tondello, Notiziario neutroni e luce di sincrotrone 12 (10) (2007) 8.

[22] http://www.elettra.trieste.it/experiments/beamlines/bear/.

[23] C.D. Wagner, W.M. Riggs, L.E. Davis, J.F. Moulder, G.E. Mullenberg, Perkin-Elmer Corp, Eden Prairie, MN, USA, 1979.

[24] J.J. Yeh, I. Lindau, At. Data Nucl. Data Tables 32 (1985) 1.

[25] M. Kasrai, W.N. Lennard, R.W. Brunner, G.M. Bancroft, J.A. Bardwell, K.H. Tan, Appl. Surf. Sci. 99 (1996) 303.

[26] A. Aquila, F. Salmassi, E. Gullikson, Opt. Lett. 33 (2008) 455.

[27] M. Suman, G. Monaco, M.-G. Pelizzo, D.L. Windt, P. Nicolosi, Opt. Express 17 (2009) 7922. 